\title{
Ancora in tema di... Scienza biomedica ed etica professionale: dalla open science al conflitto di interessi
}

\author{
Roberto Pontremoli \\ Università degli Studi e I.R.C.C.S. Azienda Ospedaliera Universitaria San Martino-IST, Istituto Nazionale per la Ricerca sul Cancro, Genova
}

\begin{abstract}
Professional ethics in biomedical science: from open access to conflict of interest
A really "open" biomedical science is an essential, widely shared asset for the progress and prosperity of modern society. Although current pathway in the communication of new scientific discoveries is good, there are gaps to be filled and areas of potential improvements, both in basic and in clinical research. These span from conflict of interest in the editorial field to the way in which new findings are conveyed to the public, and to the way in which databases from large clinical trials are analysed for the benefit of the community. However, the rapidly expanding editorial model of open access pursued by many journals may not necessarily serve the public interest because it has generated a huge amount of low quality publications without properly addressing some key issues regarding the conflict of interest.
\end{abstract}

Keywords: Open access, Conflict of interest, Peer review, Bioethical bias

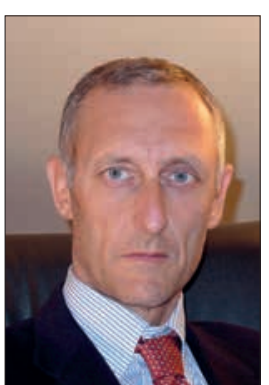

Prendo spunto dall'articolo di Duccio Lombardi (1) e dal successivo commento di Mario Timio (2), apparsi recentemente su questa rivista, per tornare, con alcune riflessioni generali, sul tema dell'etica professionale nella scienza biomedica.

\section{Open science e divulgazione nella scienza biomedica}

Roberto Pontremoli

La necessità che la scienza biomedica sia veramente open, ovvero la possibilità che tutti gli aspetti della filiera sperimentale siano disponibili innanzitutto per la comunità scientifica (destinataria naturale e in posizione privilegiata per valutarne la portata), è ormai un'esigenza ampiamente condivisa (3). In questo senso, la globalizzazione, anche informatica, degli

Accepted: August 26, 2015

Published online: September 11, 2015

Indirizzo per la corrispondenza:

Prof. Roberto Pontremoli

Università degli Studi e I.R.C.C.S.

Azienda Ospedaliera Universitaria San Martino-IST

Istituto Nazionale per la Ricerca sul Cancro

Viale Benedetto XV, 6

16132 Genova

roberto.pontremoli@unige.it ultimi due decenni ha contribuito allo sviluppo di un sistema, quello attuale, che, anche se criticabile e migliorabile sotto molti aspetti, è, tuttavia, solidamente poggiato sui principi scientifici e di democratica condivisione dei benefici per la collettività. Se, da un lato, sarebbe sbagliato invocare una rivoluzione che stravolgesse questo sistema, dall'altro persistono aree di criticità e di possibile miglioramento. Tra queste, indubbiamente, il rischio che la comunità scientifica sia vittima di vere e proprie frodi, quelle alle quali fa riferimento Lombardi, che non si limitano certo alla cosiddetta scienza di base ma che coinvolgono, spesso in maniera ancora più subdola, il settore clinico.

Come può una rivista di eccellenza come Nature difendersi dal rischio di pubblicare un lavoro fraudolento o falso? Non credo vi sia una ricetta infallibile. Spesso, è solo il "fiuto" degli Editori che può evitare pericolosi errori e la conseguente perdita di immagine e credibilità. D’altra parte, non vi è alcun dubbio sul fatto che la governance e la politica editoriale attuate dal board di una determinata rivista possano introdurre elementi di discriminazione. Per esempio, è vero che un giovane ricercatore, magari poco conosciuto, potrebbe incontrare difficoltà maggiori per comunicare i propri risultati ed è anche vero che i ricercatori established potrebbero essere, anzi spesso lo sono, indotti a occuparsi di argomenti di moda perché così è più facile pubblicare e, di conseguenza, essere, poi, finanziati. Se un lavoro sperimentale è verosimile, ci saranno sempre la possibilità e il rischio che venga pubblicato da una rivista anche prestigiosa, per poi scoprire successivamente che era falso (proprio come nel caso delle STAP cell). Ma la 
stessa metodologia scientifica ci aiuta a difenderci da queste frodi. Nei mesi e negli anni successivi alla pubblicazione, se i risultati non saranno confermati e replicati in altri laboratori indipendenti, cresceranno i sospetti e si arriverà a scoprire la frode o a ridimensionare la portata dei risultati. L'importante è che rimanga, nella comunità scientifica, una sorta di "memoria storica". II resto può essere lasciato al "mercato" cioè alla libertà dei ricercatori di replicare o meno gli studi degli altri. ॥ verdetto finale verrà, appunto, dalla comunità in senso lato, dal mercato. Non è un caso che un grande scienziato venga raramente insignito del premio Nobel nei mesi immediatamente successivi alla sua scoperta, per quanto rivoluzionaria essa sia. Più spesso questo accade dopo anni, cioè dopo che le ricadute di questa scoperta siano state ampiamente sfruttate e dopo che sia stato possibile giudicarne e apprezzarne la vera importanza. A volte, vincono il premio Nobel scoperte che, nell'immediato, erano sembrate modeste.

La ricerca clinica, però, presenta peculiarità che rendono lo scenario più complesso. L'efficacia e il successo di una terapia a volte sono oggetto di controversia tra gli stessi addetti ai lavori. Un esempio, che senz'altro è familiare ai clinici nel settore della medicina renale e cardiovascolare, è quello delle indicazioni e del rapporto rischio/beneficio della terapia di associazione con farmaci inibitori del Sistema ReninaAngiotensina. La storia è nota a tutti, ma vale la pena di ricostruirne le tappe principali. Tutto comincia circa 10 anni fa con la pubblicazione, su una prestigiosa rivista di medicina, di uno studio giapponese (4), rivelatosi falso e, successivamente, formalmente ritirato. Successivamente, viene completato un grande trial (5), i cui risultati in parte sorprendono e in parte deludono le aspettative della comunità scientifica, indotta, anche dalla pressione delle multinazionali farmaceutiche, a preconizzare una maggiore efficacia della terapia di combinazione con ACE-I/ARB rispetto alla monoterapia. A distanza di alcuni anni, nonostante due ulteriori megatrial $(6,7)$ abbiano confermato sostanzialmente la non superiorità della terapia di combinazione rispetto alla monoterapia e sebbene le Linee Guida mettano in guardia dai possibili rischi della prima, tuttavia essa è ancora largamente praticata soprattutto negli ambienti nefrologici, e autorevoli ricercatori e clinici ne declamano pubblicamente le proprietà, attribuendo ad essa un'efficacia a dir poco eccessiva nel rallentare la progressione verso I'ESRD. Qui il discorso si fa più subdolo e sottile. Non si tratta di smascherare procedure o risultati apertamente falsi ma di divulgare e diffondere interpretazioni e applicazioni dei risultati corrette e non enfatizzate, soprattutto attraverso i canali di comunicazione non scientifici. La stampa, soprattutto quella laica, ha sempre bisogno di notizie iperboliche per attrarre l'attenzione di lettori. E, alla fine, la medicina clinica, che è scienza meno "dura" della biologia, si presta maggiormente a distorsioni, quando non a vere e proprie mistificazioni. D’altra parte, come ignorare il fatto che esiste un'enorme mole di dati clinici raccolti nei vari megatrial in questi anni che rimane perlopiù non cono- sciuta e non utilizzata? Spesso, gli studi clinici finanziati o co-finanziati dall'industria farmaceutica si concentrano su un particolare aspetto, di solito terapeutico. Una volta pubblicati i risultati principali, il database non viene ulteriormente analizzato, spesso per mancanza di interesse specifico degli sponsor. Eppure, in molte aree, la nostra conoscenza, anche della semplice storia naturale di una specifica malattia, è ancora molto deficitaria, e questi dati potrebbero essere preziosi. E pensare che vi sono megatrial in campo cardiorenale che, a distanza di anni, sono stati oggetto di una sola pubblicazione scientifica! $\mathrm{E}$, allora, ben vengano iniziative internazionali come quella promossa da AllTrials (http://www. alltrials.net/), che raggruppa diverse associazioni e organizzazioni culturali e scientifiche e che si propone di favorire una maggiore trasparenza sulla gestione e sulla comunicazione dei risultati dei trial clinici.

\section{Conflitto di interessi in campo editoriale: il processo di revisione e l'open access}

$\mathrm{E}$, se tutte le riviste fossero open access, come potremmo assicurare una gerarchia qualitativa dell'informazione? La moltiplicazione delle riviste open access ha portato a una proliferazione indiscriminata di lavori sostanzialmente inutili, incontrollabili e, spesso, di scarsa valenza scientifica. E I'Accademia, almeno quella italiana, non è esente da colpe. Per esempio, la politica di valutazione della ricerca (si pensi ai criteri recentemente adottati per la valutazione dei candidati all'Abilitazione Scientifica Nazionale) ha valorizzato eccessivamente la quantità della produzione anche in modo indipendente dalla qualità. Quanto sopra porta in primo piano un altro importante aspetto strettamente collegato: il conflitto di interessi tra ricercatori e scienziati. E si tratta, come dice Timio, di un problema di non facile soluzione. È vero che il sistema dei revisori anonimi, se intaccato dal conflitto di interessi, può, in una certa misura e in alcuni casi, rallentare o, addirittura, deviare il percorso e la comunicazione della ricerca, ma esso rimane la soluzione migliore, soprattutto se si riesce a contenere il rischio di queste storture in una dimensione modesta. In altre parole, è meglio che i revisori rimangano anonimi, anche se questo comporta il rischio che alcuni non si comportino correttamente. Temo che la risposta al conflitto di interessi editoriale non possa essere il modello di business attuato da riviste totalmente open access. Queste spesso si propongono di non valutare l'importanza dell'argomento trattato da un determinato lavoro, ma si limitano, almeno in teoria, a verificarne la veridicità e la correttezza tecnica. Il costo della pubblicazione è spesso sostenuto dagli stessi Autori e il processo di revisione è, per cosi dire, "sommario". Ma spostare il costo della pubblicazione sulle spalle dell'Autore piuttosto che su quelle dei lettori equivale sostanzialmente a spostare il problema del conflitto in altra sede. Non certo a risolverlo. Gli Editori hanno interesse ad accettare e a pubblicare il numero maggiore possibile di manoscritti per massimizzare i guadagni. Non è forse un 
caso che l'impact factor di alcune riviste open access, dopo un'impennata iniziale, sia progressivamente e costantemente sceso negli anni successivi. Forse, piuttosto dell'IF della rivista, sarebbe importante verificare il numero di citazioni che un determinato lavoro riceve negli anni successivi alla sua pubblicazione. Ma anche questo indicatore non è fail-proof perché, tra autocitazioni e citazioni di convenienza e amicizia, si può gonfiare artificiosamente l'importanza di un lavoro (e, quindi, di un determinato Autore-Ricercatore).

II conflitto di interessi non si limita, poi, agli aspetti editoriali della ricerca, al fatto cioè che un editor o un revisore possano decidere le sorti di un lavoro scientifico. Esso coinvolge anche aspetti meno evidenti, ma non meno importanti, quando, per esempio, le aziende farmaceutiche affidano a parti "terze" il compito di organizzare eventi formativi ed educazionali, influenzando indirettamente la scelta degli argomenti e dei relatori. E, d'altra parte, non sono esenti da tale conflitto le organizzazioni, siano esse governative o professionali (ordi$\mathrm{ni}$, società scientifiche), quando operano in modo analogo. $\mathrm{E}$ come non pensare agli articoli indirettamente commissionati a medical writer da parte di aziende portatrici di interesse, che poi vengono pubblicati con la firma di Autori opinion leader nel campo specifico.

Open science, dunque? Certo! Ma le frodi ci saranno sempre. II sistema non sarà mai fail-proof ma, per servire in modo efficiente l'interesse della comunità, è sufficiente che il falso rimanga in percentuale minima e sostenibile, come la corruzione o il crimine in una società libera e democratica. La comunità scientifica e il metodo scientifico hanno gli strumenti per mantenere il fenomeno frode in ambito fisiologico. Certo, ci sono incidenti dannosi, soprattutto per l'immagine e per la credibilità del sistema, ma temo che affidarsi o appellarsi alla "buona fede" o alla rettitudine e alla morale dei revisori e degli Autori non sia sufficiente né accettabile. II sistema per funzionare deve avere gli strumenti intrinseci per proteggersi (e proteggerci) da questi rischi. Open science vuol dire open access? Non credo, non è così semplice. Almeno non nel senso che tutte le riviste debbano essere totalmente open. Come sarebbe possibile mantenere un ranking qualitativo dell'informazione senza una valutazione di merito che inizialmente solo la comunità culturale-scientifica può dare? Tutto questo ha un costo. Che deve essere considerato e sostenuto, se vogliamo che il sistema funzioni. Altrimenti, i più deboli, cioè il pubblico, la società, noi stessi, non potranno difendersi dalla mole soverchiante di falsità e di ciarpame che circola nell'era della comunicazione globale. A meno che non vi siano pericolose fughe in avanti. Che forse dovremmo evitare. Non dobbiamo, cioè, consentire che siano il magistrato di turno o le lobby (siano esse la rappresentazione delle multinazionali o dei consumatori-pazienti) a decidere, spesso senza cognizione di causa e sicuramente senza gli strumenti culturali e intellettuali per valutare adeguatamente se e quali terapie o interventi siano efficaci e sicuri per i cittadini o, ancora peggio, se siano eseguite a spese dello stato, cioè di tutti. Sarebbe auspicabile riuscire a identificare figure di revisori immuni dal conflitto di interessi, ma non riesco a immaginare una soluzione ottimale. È difficile che vi sia la possibilità di fare ricerca e di divulgarla senza risolvere il conflitto di interessi con i gruppi portatori di interesse, siano essi aziende, partiti politici o governi. E allora? Parafrasando Godel, potremmo dire che non esiste un sistema coerente "a prescindere"... ma, forse, nell'interesse generale della società, inclusa quella scientifica, non è neanche necessario. E un buon compromesso è la soluzione migliore.

\section{Disclosures}

Financial support: No financial support was received for this submission.

Conflict of interest: The author has no conflict of interest.

\section{Bibliografia}

1. Lombardi D. II progresso umano: quando la scienza non accresce la conoscenza. Il caso delle cellule STAP. Giornale di Tecniche Nefrologiche e Dialitiche. 2015;27:42-4.

2. Timio M. Ritorno alla bioetica della "peer review". Giornale di Tecniche Nefrologiche e Dialitiche. 2015;27(1):1-3.

3. Pelliccia F, Coats AJ, Pani L, Gaudio C, Rosano G. Transparency in medical research: Time for a paradigm shift. Int J Cardiol. 2015;186:259-60.

4. Nakao N, Yoshimura A, Morita H, Takada M, Kayano T, Ideura T. Combination treatment of angiotensin-II receptor blocker and angiotensin-converting-enzyme inhibitor in non-diabetic renal disease (COOPERATE): a randomised controlled trial. Lancet. 2003;361(9352):117-24.

5. Mann JF, Schmieder RE, McQueen M, et al. Renal outcomes with telmisartan, ramipril, or both, in people at high vascular risk (the ONTARGET study): a multicentre, randomised, doubleblind, controlled trial. Lancet. 2008;372(9638):547-53.

6. Parving $\mathrm{HH}$, Brenner BM, McMurray JJ, et al. Cardiorenal end points in a trial of aliskiren for type 2 diabetes. N Engl J Med. 2012;367(23):2204-13.

7. Fried LF, Emanuele $\mathrm{N}$, Zhang $\mathrm{JH}$, et al. Combined angiotensin inhibition for the treatment of diabetic nephropathy. N Engl J Med. 2013;369(20):1892-903. 